\title{
Editorial issue 2/3 2018
}

\author{
Maurits W. Ertsen ${ }^{1} \cdot$ Ellen F. Arnold ${ }^{2}$
}

Published online: 28 September 2018

(C) Springer Nature B.V. 2018

In this double issue, numbers 2 and 3 of 2018, we are happy to present papers offering new ideas in terms of areas that we study and methods that we use to develop a fuller picture of water history.

The first two papers bring us to the Hellenistic water world in very different ways. In "Running water for the officials, rainwater for the poor", Yannis Spyropoulos discusses water management on Crete between 1645 and 1830. In this detailed analysis of challenges faced by administration and population on an island with limited water resources, our attention is redirected from heavily-populated cities and plains to smaller regions. In their contribution, Patrik Klingborg and Martin Finné present their modelling efforts on rainwater harvesting cisterns of Olynthos in Thessaly and Dystos on ancient Euboia-both in ancient Greece. Their results suggest that cisterns could provide freshwater to households and act as buffers in shorter (monthly), and longer terms (seasonally, yearly and between years) of water stress. Their work explains the efficacy of cistern systems by bringing together the predictable variability of water availability and active participation from members of the household.

In the second portion of this double issue, we welcome guest editors Nicolas Maughan, Alexey Kraikovski and Julia Lajus. Their set of five papers has a separate editorial introduction about urban water, with specific attention for the evolution of both water supply systems and wastewater technologies through the centuries. These articles bring us to Venice, Sint Petersburg, Versailles, and late Imperial and Soviet Russia (Lake Baikal and River Vuoksi). Together, the papers present new developments in histories of urban water. We are very happy to publish the results of what started as a series of environmental history workshops dealing with the history of urban landscapes and water management held in the European University at St. Petersburg and Venice, plus during thematic sessions of ESEH, ASEH and IWHA Conferences.

Maurits W. Ertsen

M.W.Ertsen@tudelft.nl

1 Water Resources Management, Delft University of Technology, Delft, The Netherlands

2 History, Ohio Wesleyan University, Delaware, USA 